\title{
Smart glasses display device for fluoroscopically guided minimally invasive spinal instrumentation surgery: a preliminary study
}

\author{
Keitaro Matsukawa, MD, PhD, and Yoshiyuki Yato, MD, PhD \\ Department of Orthopaedic Surgery, National Hospital Organization, Murayama Medical Center, Musashimurayama, Tokyo, \\ Japan
}

\begin{abstract}
OBJECTIVE Most surgeons are forced to turn their heads away from the surgical field to see various intraoperative support monitors. These movements may result in inconvenience to surgeons and lead to technical difficulties and potential errors. Wearable devices that can be attached to smart glasses or any glasses are novel visualization tools providing an alternative screen in front of the user's eyes, allowing surgeons to keep their attention focused on the operative task without taking their eyes off the surgical field. The aim of the present study was to examine the feasibility of using glasses equipped with a wearable display device that transmits display monitor data during fluoroscopically guided minimally invasive spinal instrumentation surgery.
\end{abstract}

METHODS In this pilot prospective randomized study, 20 consecutively enrolled patients who underwent singlesegment posterior lumbar interbody fusion (PLIF) at L5-S1 performed using the percutaneous pedicle screw technique were randomly divided into two groups, a group for which the surgeon used a wearable display device attached to regular glasses while performing surgery (smart glasses group) and a group for which the surgeon did not use such a device (nonglasses group). Real-time intraoperative fluoroscopic images were wirelessly transmitted to the display device attached to the surgeon's glasses. The number of head turns performed by the surgeon to view the standard fluoroscopic monitor during procedures and the operative time, estimated blood loss, radiation exposure time, screw placement accuracy, and intraoperative complication rate were evaluated for comparison between the two groups.

RESULTS The number of surgeon head turns to view the fluoroscopic monitor in the smart glasses group was $0.10 \pm$ 0.31 times, which was significantly fewer than the head turns in the nonglasses group ( $82.4 \pm 32.5$ times; $p<0.001)$. The operative and radiation exposure times in the smart glasses group were shorter than those in the nonglasses group (operative time $100.2 \pm 10.4$ vs $105.5 \pm 14.6$ minutes, radiation exposure time $38.6 \pm 6.6$ vs $41.8 \pm 16.1$ seconds, respectively), although the differences were not significant. Postoperative CT showed one screw perforation in the nonglasses group, and no intraoperative complications were observed in either group.

CONCLUSIONS This is, to the authors' knowledge, the first report on the feasibility of using this wearable display device attached to glasses for fluoroscopically guided minimally invasive spinal instrumentation surgery. Smart glasses display devices such as this one may be a valid option to facilitate better concentration on operative tasks by improving ergonomic efficiency during surgery.

https://thejns.org/doi/abs/10.3171/2020.6.SPINE20644

KEYWORDS smart glasses; head-mounted display; fluoroscopy-guided; minimally invasive; spine instrumentation; surgical technique

$\mathrm{V}$ ARIOUS intraoperative support devices, such as vital sign monitors, fluoroscopic guidance, navigation systems, and neurophysiological monitoring, are essential to ensure patient safety and avoid complications during spine surgery. Surgeons are required to collectively consider information from these multiple audiovisual devices, which assists them in making judgments leading to successful completion of the surgery. This trend has become more pronounced in recent years with the rapidly increasing use of minimally invasive spine surgery. In order to achieve the same accuracy as conventional open surgery in a narrow and limited surgical field, surgeons use various intraoperative support devices that can replace or enhance the operator's eyes and are now indispensable.

ABBREVIATIONS HDMI = high-definition multimedia interface; PLIF = posterior lumbar interbody fusion.

SUBMITTED April 21, 2020. ACCEPTED June 1, 2020.

INCLUDE WHEN CITING Published online October 13, 2020; DOI: 10.3171/2020.6.SPINE20644. 


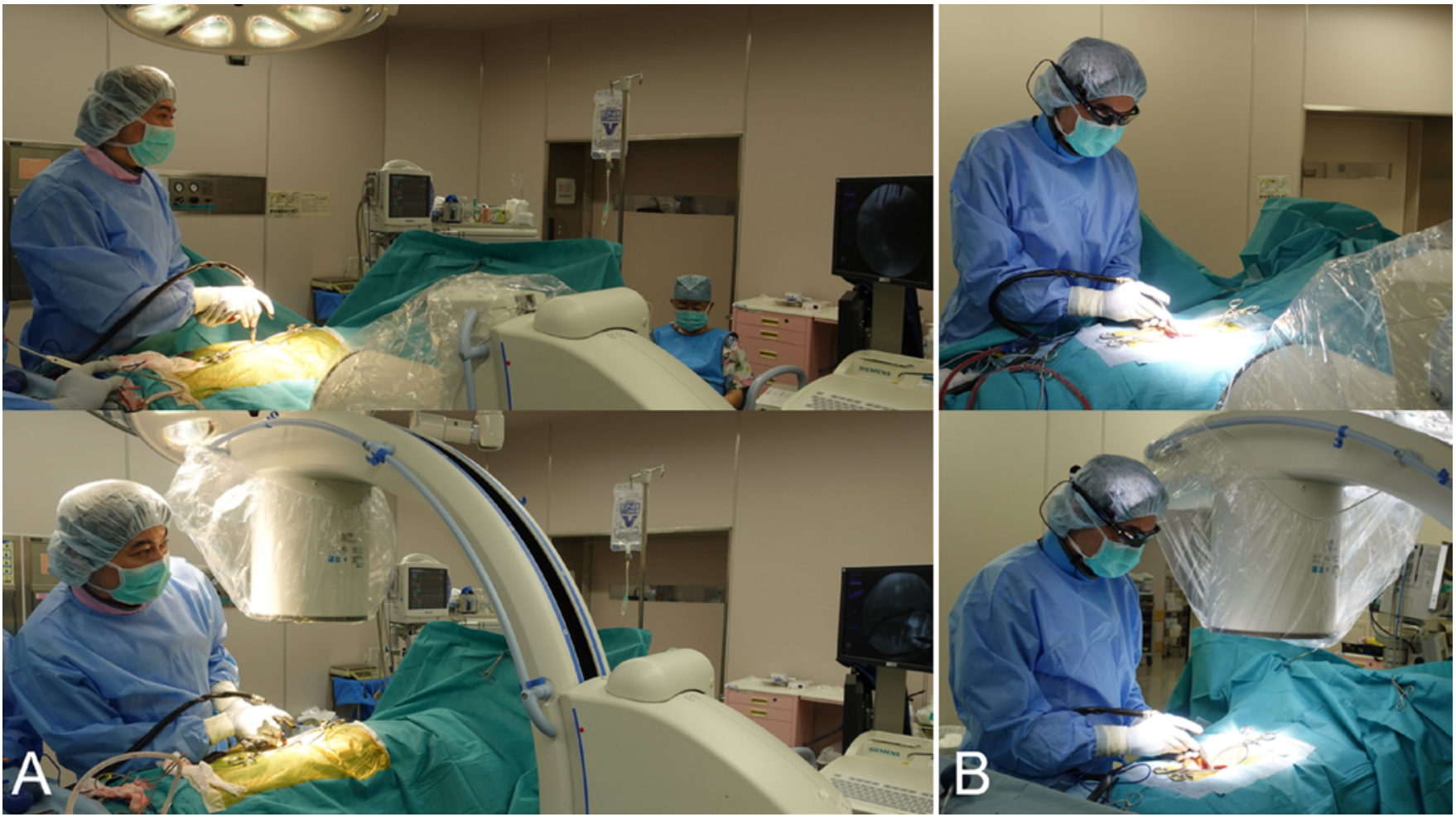

FIG. 1. Comparison of fluoroscopy-guided procedures. A: The surgeon views the standard fluoroscopic monitor by turning the head away from the surgical field. B: The surgeon uses the smart glasses display device as an alternative method to display fluoroscopic images, and so the surgeon's focus can be maintained on the surgical field. Figure is available in color online only.

There are two major problems with this surgical environment. First, these monitors are not always located in positions where they can be easily viewed by surgeons or assistants, because of cumbersome equipment within limited-capacity operating rooms. Second, to check the monitors during a delicate spinal procedure, surgeons are forced to turn their head away and take their eyes off the surgical field, in some cases twisting their body or removing it from the surgical field (Fig. 1A). These unnecessary movements may result in inconvenience to surgeons and cause technical difficulties and errors. For example, during percutaneous pedicle screw placement, surgeons determine the insertion points, place needles at the appropriate depth, and insert cannulated pedicle screws while frequently checking an intraoperative fluoroscopic monitor to avoid displacing wires. Simultaneously, surgeons can confirm the correctness of screw placement by checking a neuromonitor. Such frequent eye movements between the surgical field and monitors interfere with the smooth progression of surgery, potentially leading to unintentional hand deviation and critical misplacement, a longer operative time, and unnecessary radiation exposure. This tendency is marked in inexperienced surgeons.

Portable head-mounted display devices that present information directly in the user's visual field and promote hands-free capabilities have been applied in medical and healthcare fields in recent years. ${ }^{1}$ These devices are divided into two major categories, non-see-through displays obscuring the user's field of view and see-through displays showing information overlaid onto the user's field of view. Compact, lightweight, and comfortable head-mounted displays are now available that can be attached to any type of glasses. By superimposing various kinds of intraoperative information projected on the display within the user's visual field, device-assisted "smart" glasses allow surgeons to keep their attention focused on the operative task without taking their eyes away from the surgical field (Fig. 1B). In the literature, some authors have reported the advantages of using smart glasses in terms of improved accuracy and reduced time during various surgical procedures. ${ }^{2-6} \mathrm{Al}-$ though minimally invasive surgery has become a popular technique and offers many benefits to both surgeons and patients, these techniques depend heavily on indirect visualization and fluoroscopic navigation guidance. We hypothesized that the utilization of device-assisted glasses for the placement of minimally invasive spinal instrumentation might improve the safety of the surgery. However, to the best of our knowledge, little has been reported on the use of a device attached to surgical glasses in spine surgery for monitoring surgical data projected in the surgeon's field of view. The aim of the present study was to examine the feasibility of using a smart glasses display device for fluoroscopy-guided posterior lumbar interbody fusion (PLIF) involving the percutaneous pedicle screw technique.

\section{Methods}

This was a pilot prospective randomized study conducted from April 2019 to March 2020. The study participants 


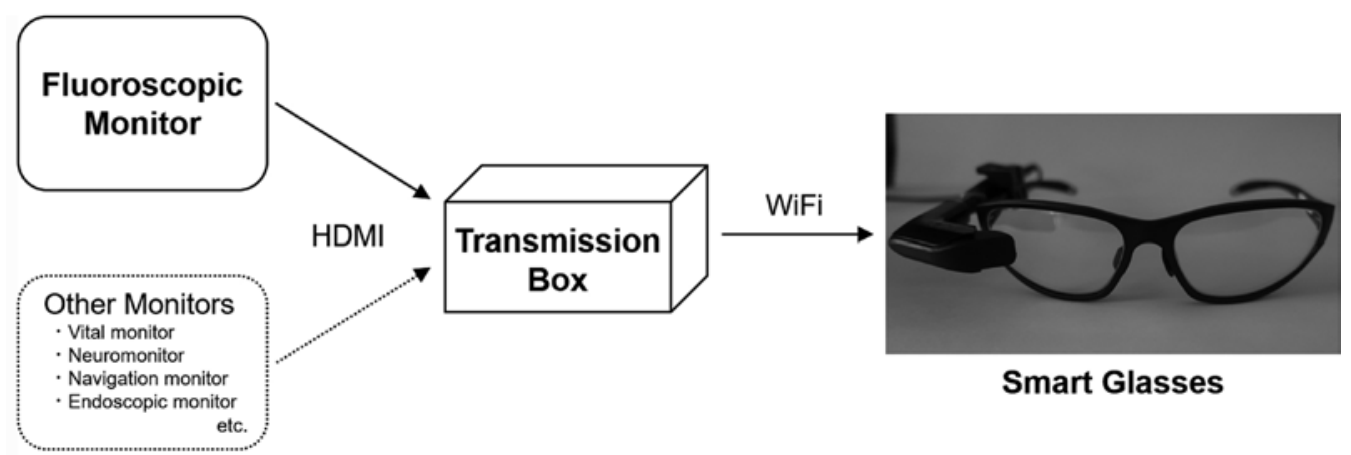

FIG. 2. Image capture and streaming system using the smart glasses display device. Intraoperative information (only fluoroscopic images in this study) is output to a transmission box using an HDMI signal, which is transmitted to the picoLinker device via WiFi.

consisted of 20 consecutively enrolled patients (15 men and 5 women; mean age $61.9 \pm 12.5$ years, range $42-82$ years) who underwent single-segment PLIF at L5-S1. All 20 operations were performed by the same surgeon, who used the percutaneous pedicle screw technique. There were 15 patients with spondylolytic spondylolisthesis, four with recurrent disc herniation, and one with foraminal stenosis. Patients were randomly divided into two groups in a 1:1 allocation, one group in which the surgeon used device-assisted smart glasses to perform the PLIF (smart glasses group) and another group in which the surgeon did not use a smart glasses device (nonglasses group). Randomization was achieved by an independent observer who used the sealed envelope method.

\section{Image Capture and Streaming System Using the picoLinker Device}

Intraoperative fluoroscopically guided procedures were conducted using an Arcadis Orbic 3D fluoroscopy device (Siemens). First, real-time intraoperative fluoroscopic images were output to a transmission box using a high-definition multimedia interface (HDMI) signal (Fig. 2). Then, this information was wirelessly transmitted to a wearable display device attached to the surgeon's glasses, which displayed information in the surgeon's field of view, using an encrypted connection via WiFi (Miracast). The time needed for image transmission was about 0.2 seconds, which was fast enough for clinical use. A commercially available monocular see-through head-mounted display was used in this study (picoLinker, Westunitis) (Fig. 3). The picoLinker is lightweight (30 g) and its flexible arm and connector clip can be easily attached to any type of glasses, including a surgical loupe, ${ }^{7}$ which allows free adjustment to the user's preferred position and quick removal if necessary due to discomfort during surgery. The picoLinker has more than 10 hours of battery life and a transparent prism screen with resolution of $640 \times 360$ pixels. The display is usually positioned in the right upper quadrant of the user's right eye and renders images equivalent to viewing a 23inch screen at a distance of $2 \mathrm{~m}$ in front of the user.

\section{Surgical Procedure}

The same PLIF procedures were performed in all patients, including bilateral total facetectomy, intervertebral discectomy, local bone grafting, and placement of two rectangular interbody cages, followed by bilateral percu-
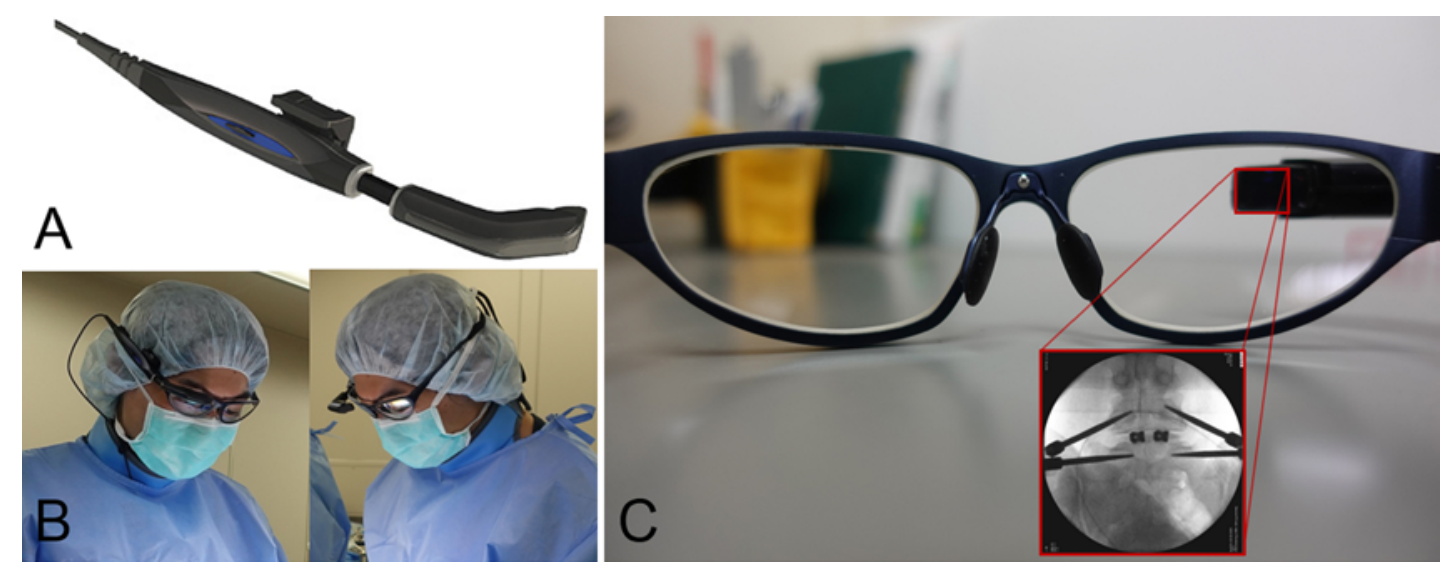

FIG. 3. The picoLinker device used in this study. A: The device can be easily attached to any type of glasses using its flexible arm and connector clip. Copyright Keitaro Matsukawa. Used with permission. B: The surgeon wearing the smart glasses device during surgery. C: The smart glasses display device and a projected intraoperative fluoroscopic image. Figure is available in color online only. 
TABLE 1. Summary of patient characteristics $(n=20)$

\begin{tabular}{|c|c|c|c|}
\hline & \multicolumn{2}{|c|}{ Patient Group } & \multirow[b]{2}{*}{ p Value } \\
\hline & Smart Glasses & Nonglasses & \\
\hline Mean age, yrs & $60.8 \pm 15.9$ & $63.1 \pm 8.4$ & 0.69 \\
\hline Mean sex, M:F & 8:2 & $7: 3$ & $>0.99$ \\
\hline Mean BMI, kg/m² & $24.5 \pm 2.5$ & $24.3 \pm 2.5$ & 0.73 \\
\hline Diagnosis & & & $>0.99$ \\
\hline $\begin{array}{l}\text { Spondylolytic } \\
\text { spondylolisthesis }\end{array}$ & 8 & 7 & \\
\hline Recurrent disc herniation & 2 & 2 & \\
\hline Foraminal stenosis & 0 & 1 & \\
\hline
\end{tabular}

Values are presented as number of patients or mean \pm SD.

taneous pedicle screw fixation. Fluoroscopic guidance was typically used for the following procedures: preoperative skin marking, cage placement, needle placement, tapping and screw insertion, bilateral rod placement, and reduction maneuvers as needed. In the glasses group, the surgeon utilized the picoLinker smart glasses system during all process mentioned above, whereas in the nonglasses group, the surgeon utilized a standard fluoroscopic monitor.

\section{Evaluation}

The number of times the surgeon's head turned to view the fluoroscopic monitor during procedures and the operative time, estimated blood loss, radiation exposure time, accuracy of screw placement, and any intraoperative complications were evaluated for comparison between the two groups. Screw positions were assessed in CT images obtained within 1 week postoperatively, and screw perforation was defined as a screw with any cortical breach.

\section{Statistical Analysis}

All results are shown as the mean \pm standard deviation. Student and Welch t-tests were used for continuous variables, and Fisher exact tests were used for categorized variables. JMP version 13 (SAS Institute) was used for all analyses, and the significance was set at $\mathrm{p}<0.05$.

\section{Results}

There were no significant differences in patient demographic or medical characteristics, such as age, sex, body mass index, and diagnosis, between the two groups (Table 1). The number of surgeon head turns to view the fluoroscopic monitor in the smart glasses group was $0.10 \pm 0.31$ times (range $0-1$ times), which was significantly fewer than in the nonglasses group $(82.4 \pm 32.5$ times, range 54-132 times; $\mathrm{p}<0.001$ ) (Table 2). The operative and radiation exposure times in the smart glasses group were shorter than those in the nonglasses group (operative time $100.2 \pm 10.4$ vs $105.5 \pm 14.6$ minutes, radiation exposure time $38.6 \pm 6.6$ vs $41.8 \pm 16.1$ seconds, respectively), although the differences were not significant. Postoperative CT showed one screw in the nonglasses group with a medial breach of less than $2 \mathrm{~mm}$. There were no other com-
TABLE 2. Summary of overall results

\begin{tabular}{lccc}
\hline & \multicolumn{2}{c}{ Patient Group } & \\
\cline { 2 - 3 } & Smart Glasses & Nonglasses & p Value \\
\hline No. of head turns & $0.10 \pm 0.31$ & $82.4 \pm 32.5$ & $<0.001$ \\
\hline Operative time, mins & $100.2 \pm 10.4$ & $105.5 \pm 14.6$ & 0.36 \\
\hline Estimated blood loss, $\mathrm{mL}$ & $55.1 \pm 35.1$ & $66.3 \pm 38.3$ & 0.51 \\
\hline Radiation exposure time, sec & $38.6 \pm 6.6$ & $41.8 \pm 16.1$ & 0.57 \\
\hline Screw perforation, no. $(\%)$ & $0 / 40(0 \%)$ & $1 / 40(2.5 \%)$ & $>0.99$ \\
\hline
\end{tabular}

Values are presented as mean \pm SD unless otherwise indicated.

plications, such as intraoperative dural injury, postoperative hematoma, or infection, in any of the other patients in both groups.

\section{Discussion}

The present study is, to our knowledge, the first to investigate the feasibility of using display device-assisted smart glasses during fluoroscopy-guided minimally invasive spine instrumentation surgery. The device-assisted visualization technology, which can be attached to regular glasses to display information in the user's field of view, allowed surgeons to proceed with the surgery without having to take their focus away from procedures, and thus this method has the potential to facilitate surgeon viewing of monitoring processes and reduce radiation exposure.

Head-mounted display technology with medical applications has been expanding in the current decade. Kaneko et al. and Lim et al. examined the merits of using smart glasses during an ultrasound-guided procedure in their randomized control studies, and these investigators concluded that smart glasses improved ergonomic efficiency by reducing head movements. ${ }^{4,6}$ In the field of neurospine surgery, several attempts to use smart glasses displays as an intraoperative neuromonitor, ${ }^{8}$ endoscopic monitor, ${ }^{9}$ or $3 \mathrm{D}$ navigation screen have been reported. Yoon et al. investigated the feasibility of streaming navigation images onto smart glasses during pedicle screw placement. ${ }^{3}$ According to their report, time per screw placement was $15.1 \%$ shorter when using smart glasses, with a user positive response rate of $79 \%$. The results of the present study showed similar trends in terms of shorter operative and radiation exposure times (not significant). The small sample size and operator's familiarity with the minimally invasive spine procedure could have a marked impact on the results to determine whether smart glasses display device-assisted visualization technology is of merit for use in pedicle screw placement.

In the lumbosacral region, due to the large volume of the paraspinal muscles, deeper screw entry points, and large medialized pedicle axis, the percutaneous pedicle screw technique is particularly effective compared with the conventional technique, which requires extensive muscle dissection for screw insertion. In addition, from anatomical standpoints, the sacral vertebrae have few prominent structures, unlike the transverse process of the lumbar vertebrae that can be used to identify entry points, and S1 screw insertion into the sacral promontory requires 
skillful use of fluoroscopic assistance. Consequently, particularly in this region, frequent fluoroscopic checks are required to appropriately place screws. Therefore, the present study included only patients who underwent PLIF at the L5-S1 segment.

The results of the present study have practical implications in terms of ergonomic improvements for surgeons during procedures, because intraoperative support monitors are not in line with the procedural site or the surgeons' natural line of sight. Movement of the surgeon's head away from the surgical field could lead to hand deviation, resulting in changes in position, direction, or depth within the surgical field. However, using the smart glasses system as an alternative screen in the normal field of view allows surgeons to see both the surgical field and the information displayed on monitors simultaneously without moving their eyes and head. In addition to fluoroscopic images, a variety of images, such as images obtained before surgery and those for monitoring vital signs ${ }^{10}$ and neurophysiological indicators, ${ }^{8}$ conducting computer navigation, ${ }^{3}$ and endoscopic viewing, can be displayed on the wearable screen (Fig. 2). ${ }^{9}$ When using a wireless connection, surgeons can perform procedures more comfortably with full-body mobility.

Furthermore, this novel visualization approach provides two potential advantages alongside facilitating better concentration on the operative task. First, the device-assisted smart glasses system could contribute to shortening the operative time and reducing intraoperative radiation exposure. Chimenti and Mitten ${ }^{2}$ and Tsubosaka et al. ${ }^{5}$ performed a study involving fluoroscopy-guided pin insertion for hand and femoral neck fracture and demonstrated that the use of smart glasses reduced the operative time by 15\%-33\% and intraoperative radiation exposure time by 44\%-59\% compared with a control. In the present study, the use of a smart glasses display device (picoLinker) also seemed to reduce unintentional hand deviation during procedures by eliminating the need to shift the focus between a standard monitor and the surgical field, leading to shorter operative and radiation exposure times. Second, equipped with a camera and video-recording capabilities, this new device could become a more useful adjunct tool in surgical education, information sharing, real-time consultation, and remote instruction and monitoring.,11,12

There are some concerns to be noted, such as the wearability of and fatigue caused by using a smart glasses device. We did not conduct a survey to assess feedback from multiple users on the usability and comfort of wearing such a device attached to glasses. However, the surgeon in this study experienced no discomfort or adverse effects and there was no significant increase in the operative time, possibly owing to the lightweight body construction of the device, adjustability to the user's preferred position, and transparent monocular display that did not interfere with performing tasks. Our study included only patients undergoing single-segment fusion; thus, further research on the effects of prolonged use in more complex cases is needed.

Several limitations of this study should be acknowledged. First, this study presented the initial experience of using the display device-assisted smart glasses; however, the presence of a learning curve for the use of this system was not investigated. Different conclusions may be drawn depending on the degree of the surgeon's familiarity with the use of glasses with an additional visual screen provided by an attached device. Second, we investigated only the radiation exposure time and lacked data on radiation dose to surgeons and the number of images taken during fluoroscopy. Because the radiation dose is affected not only by the time of exposure, but also by the distance from the radiation source and shielding devices, further studies to assess the effective dose of fluoroscopic radiation are warranted. Third, we evaluated the total operative time instead of the time taken for each individual procedure, such as skin marking, cage placement, and screw placement. The placement of percutaneous pedicle screws is one of the procedures that heavily depends on fluoroscopic assistance; therefore, an assessment of the time taken for screw placement may be useful to elucidate in detail the true benefits of using smart glasses.

\section{Conclusions}

To the best of our knowledge, this is the first report on the feasibility of using device-assisted smart glasses for fluoroscopy-guided minimally invasive spinal instrumentation placement. The use of a smart glasses display device such as this one may be a valid option to enhance the surgeon's ability to concentrate on operative tasks by improving ergonomic efficiency during surgery.

\section{References}

1. Rahman R, Wood ME, Qian L, et al. Head-mounted display use in surgery: a systematic review. Surg Innov. 2020;27(1): 88-100.

2. Chimenti PC, Mitten DJ. Google Glass as an alternative to standard fluoroscopic visualization for percutaneous fixation of hand fractures: a pilot study. Plast Reconstr Surg. 2015; 136(2):328-330.

3. Yoon JW, Chen RE, Han PK, et al. Technical feasibility and safety of an intraoperative head-up display device during spine instrumentation. Int J Med Robot. 2017;13(3):e1770.

4. Kaneko N, Tsunoda M, Mitsuhashi M, et al. Ultrasoundguided fine-needle aspiration in the neck region using an optical see-through head-mounted display: a randomized controlled trial. J Ultrasound Med. 2017;36(10):2071-2077.

5. Tsubosaka M, Hiranaka T, Okimura K, et al. Additional visualization via smart glasses improves accuracy of wire insertion in fracture surgery. Surg Innov. 2017;24(6):611-615.

6. Lim H, Kim MJ, Park JM, et al. Use of smart glasses for ultrasound-guided peripheral venous access: a randomized controlled pilot study. Clin Exp Emerg Med. 2019;6(4):356-361.

7. Diaz R, Yoon J, Chen R, et al. Real-time video-streaming to surgical loupe mounted head-up display for navigated meningioma resection. Turk Neurosurg. 2017;28(4):682-688.

8. Golab MR, Breedon PJ, Vloeberghs M. A wearable headset for monitoring electromyography responses within spinal surgery. Eur Spine J. 2016;25(10):3214-3219.

9. Liounakos JI, Urakov T, Wang MY. Head-up display assisted endoscopic lumbar discectomy-a technical note. Int J Med Robot. 2020;16(3):e2089.

10. Iqbal MH, Aydin A, Lowdon A, et al. The effectiveness of Google GLASS as a vital signs monitor in surgery: a simulation study. Int J Surg. 2016;36(Pt A):293-297.

11. Guze PA. Using technology to meet the challenges of medical education. Trans Am Clin Climatol Assoc. 2015;126: 260-270. 
12. Rojas-Muñoz E, Cabrera ME, Lin C, et al. The System for Telementoring with Augmented Reality (STAR): a headmounted display to improve surgical coaching and confidence in remote areas. Surgery. 2020;167(4):724-731.

\section{Disclosures}

Keitaro Matsukawa is a consultant for Westunitis.

\section{Author Contributions}

Conception and design: Matsukawa. Acquisition of data:

Matsukawa. Analysis and interpretation of data: Matsukawa.

Drafting the article: Matsukawa. Critically revising the article:

Yato. Reviewed submitted version of manuscript: Yato. Approved the final version of the manuscript on behalf of both authors: Matsukawa. Statistical analysis: Matsukawa. Administrative/ technical/material support: Matsukawa. Study supervision: both authors.

\section{Correspondence}

Keitaro Matsukawa: National Hospital Organization, Murayama Medical Center, Tokyo, Japan. keitaro197897@hotmail.com. 